\title{
Identification of key differentially expressed genes and gene mutations in breast ductal carcinoma in situ using RNA-seq analysis
}

\author{
Congyuan Zhu*, Hao Hu, Jianping Li*, Jingli Wang, Ke Wang and Jingqiu Sun
}

\begin{abstract}
Background: The aim of this study was to identify the key differentially expressed genes (DEGs) and high-risk gene mutations in breast ductal carcinoma in situ (DCIS).

Methods: Raw data (GSE36863) were downloaded from the database of Gene Expression Omnibus (GEO), including three DCIS samples (DCIS cell lines MCF10.DCIS, Sum102, and Sum225) and one normal control sample (normal mammary epithelial cell line MCF10A). The DEGs were analyzed using NOlseq and annotated via DAVID. Motif scanning in the promoter region of DEGs was performed via SeqPos. Additionally, single nucleotide variations (SNVs) were identified via GenomeAnalysisTK and SNV risk was assessed via VarioWatch. Mutant genes with a high frequency and risk were validated by RT-PCR analyses.

Results: Finally, 5391, 7073, and 7944 DEGs were identified in DCIS, Sum102, and Sum22 cell lines, respectively, when compared with MCF10A. VENN analysis of the three cell lines revealed 603 upregulated and 1043 downregulated DEGs, including 16 upregulated and 36 downregulated transcription factor (TF) genes. In addition, six TFs each (e.g., E2F1 and CREB1) were found to regulate the core up- and downregulated DEGs, respectively. Furthermore, SNV detection results revealed 1104 (MCF10.DCIS), 2833 (Sum102), and 1132 (Sum22) mutation sites. Four mutant genes (RWDD4, SDHC, SEPT7, and SFN) with high frequency and risk were identified. The results of RTPCR analysis as well as bioinformatics analysis consistently demonstrated that the expression of RWDD4, SDHC, SEPT7, and SFN was downregulated in the tumor tissues as compared with that in adjacent non-tumor tissues.

Conclusions: The differentially expressed TFs, TFs regulating DEGs (e.g., E2F1 and CREB1), and high-frequency mutant genes (RWDD4, SDHC, SEPT7, and SFN) might play key roles in the pathogenesis of DCIS.
\end{abstract}

Keywords: Breast ductal carcinoma in situ, Differentially expressed genes, Mutant genes, RNA-Seq analysis, Transcription factor

\section{Background}

Breast ductal carcinoma (BDC), a type of breast cancer, is caused by disordered endocrine function in the ovarian tissue [1]. In 2014, there were 252,710 estimated new breast cancer cases and 40,610 deaths caused by breast

\footnotetext{
*Correspondence: brlx|2001@126.com; brlx|2001@126.com

Department of General Surgery, the Affiliated Hospital of Jiangnan University (original area of Wuxi Third People's Hospital), No. 585 North Xingyuan Road, Liangxi District, Wuxi 214002, Jiangsu, China
}

cancer in the USA [2]. Ductal carcinoma in situ (DCIS) has become a common disease mostly affecting those in their sixth decade of life, accounting for $20-25 \%$ of all incident breast malignancies in industrialized countries [3]. DCIS is histologically characterized by the proliferation of malignant epithelial cells around the basement membrane of the mammary ducts [4]. It is becoming increasingly important to shed light on the molecular mechanisms underlying DCIS.

(c) The Author(s). 2020 Open Access This article is licensed under a Creative Commons Attribution 4.0 International License, which permits use, sharing, adaptation, distribution and reproduction in any medium or format, as long as you give appropriate credit to the original author(s) and the source, provide a link to the Creative Commons licence, and indicate if changes were made. The images or other third party material in this article are included in the article's Creative Commons licence, unless indicated otherwise in a credit line to the material. If material is not included in the article's Creative Commons licence and your intended use is not permitted by statutory regulation or exceeds the permitted use, you will need to obtain permission directly from the copyright holder. To view a copy of this licence, visit http://creativecommons.org/licenses/by/4.0/ The Creative Commons Public Domain Dedication waiver (http://creativecommons.org/publicdomain/zero/1.0/) applies to the data made available in this article, unless otherwise stated in a credit line to the data. 
Remarkable developments in genomic technologies have made researchers decipher the genetic changes that occur in cancer. For example, RBBP7 (RbAp46) and BIRC5 (survivin) expression in carcinoma cells is significantly higher in estrogen receptor (ER)-positive pure DCIS than in invasive ductal carcinoma (IDC) [5]. Genes involved in cell signaling and adhesion (TMEM45A, FAT1, and DST) in DCIS have been found to be closely correlated with the transition from in situ cancer to invasive cancer [6]. Furthermore, gene mutations in DCIS have also been associated with the progression of DCIS; these include BRCA1/2 deleterious mutation [7], somatic mutations in AKT1, PIK3CA, and TP53 [8], and hypermethylation of HOXA5 and SOX genes [9].

In recent years, next-generation sequencing-based approaches, such as RNA-Sequencing (RNA-Seq), have garnered the potential to offer unprecedented in-depth analysis of gene expression. Based on RNA-Seq analysis, Tian et al. [10] identified differentially expressed transcripts in DCIS models, which are associated with signaling pathways such as cell proliferation, cell-cell adhesion, and cell-cell signaling, and reported that aldehyde dehydrogenase 5A1 (ALDH5A1) may serve as a novel molecular target in DCIS treatment. However, genetic mutations associated with DCIS were not investigated. In the present study, RNA-Seq data deposited by Kaur et al. [11], including three DCIS samples (MCF10.DCIS, Sum102, and Sum22) and one normal control sample (MCF10A), were downloaded to identify the differentially expressed genes (DEGs). After functional annotation of the DEGs, motif finding was performed in the promoter region of DEGs. Additionally, single nucleotide variants (SNVs) in DCIS were identified and annotated. These results might improve our understanding of the molecular mechanism underlying DCIS and suggest potential targets for clinical treatment.

\section{Materials and methods \\ Data sources of RNA-seq}

The RNA-seq data GSE36863 [11] were downloaded from the database of Gene Expression Omnibus (GEO), including three DCIS samples (DCIS cell lines MCF10.DCIS (GSM903304), Sum102 (GSM903305), and Sum22 (GSM903306)), and one normal control sample (mammary epithelial cell MCF10A (GSM903303)). The samples were subjected to 76 cycles of single-end sequencing via an Illumina Genome Analyzer GAIIx (Illumina Inc., San Diego, California, USA). Two biological replicates of the three DCIS models (Sum102, MCF10.DCIS, and Sum225) and the MCF10A model were used to perform whole transcriptome sequencing.

MCF10.DCIS and MCF10A cell lines were obtained from the Cell Lines Resource (Karmanos Cancer Institute, Detroit, MI). Sum225 and Sum102 cell lines were provided by Dr. Stephen Ethier (Hollings Cancer Center,
Charleston, SC). Sum225 cells were isolated from a chest wall recurrence in a DCIS patient. The Sum102 cell line was isolated from a patient who was diagnosed with extensive DCIS with areas of micro-invasion. The four cell lines were grown in three dimensional (3D) reconstituted basement membrane (rBM) overlay culture for 12 days and the culture medium was changed every 4 days.

\section{Read alignment and differential expression analysis}

All the RNA-seq data were mapped to the reference human genome (hy19) in University of California Santa Cruz database using TopHat [12]. For each read, mismatches $\leq$ two bases were permitted, and other parameters followed the default settings. After read alignment, on the basis of the gene annotation data in the reference sequence (Refseq) database, the transcripts were assembled using Cufflinks [13], and the gene expression levels of the transcripts were calculated using fragments/kilobase/million reads method in Cuffdiff [13]. DEGs between DCIS and normal samples were then selected via NOISeq [14] with a $q$ value $\geq 0.8$.

\section{Functional annotation of DEGs}

The Database for Annotation, Visualization and Integrated Discovery (DAVID) [15] was used to analyze the Gene Ontology (GO) functions, including biological process (BP), molecular function (MF), and cellular component (CC), of DEGs. A $p$ value $<0.05$ was set as the criterion. Then, DEGs with transcription factor (TF) functions were screened and annotated based on the TRANSFAC database (http://www.genomatix. de). Ultimately, according to the tumor suppressor genes (TSGs) in the TSgene database [16] and the tumor associated genes (TAGs) [17], known TSGs, and oncogenes were identified in the DEGs.

\section{Examination of upstream regulatory element of DEGs}

The VENN test was conducted to select the co-expressed DEGs in the MCF10.DCIS, Sum102, and Sum225 cell lines. In the promoter region (from $1 \mathrm{~kb}$ upstream to $0.5 \mathrm{~kb}$ downstream of transcription initial site) of the up- and downregulated DEGs, motif finding was performed using Seqpos [18] to predict their potential TFs. A $p$ value $<0.00001$ and frequency $>50 \%$ were set as cutoff criteria.

\section{SNV detection}

SNVs were detected using GenomeAnalysisTK [19] with the criteria of coverage $>5$ and quality score $\geq 30$. Especially, SNVs with a quality score of $>50$ were defined as the high-reliability SNVs. On the basis of the known single nucleotide polymorphisms (SNPs) recorded in the dbSNP137 database and 1000 Genomes Project, the known SNPs in the SNVs were removed. Besides, SNV calling was optimized by using the RNA-seq data of the normal sample, and accordingly, RNA editing in the transcriptome was removed effectively. 


\section{Annotation of somatic mutant sites and detection of high-risk mutation}

VarioWatch can provide the annotations of two human genome reference versions (NCBI build 36.3, NCBI build 37.2), including gene annotations, known variants from dbSNP, pre-computed variation risks, 1000 Genomes Project (released on October 2011), OMIM (T), and other minor variant databases [20]. In this study, VarioWatch was applied to annotate the SNVs in the coding regions of the mutant genes and analyze their corresponding influence on the protein products. The genes with high-risk base mutations in each sample, and their frequency of occurrence in the three cell lines, were also analyzed. The mutations that occurred in more than 2 cell lines were defined as high-frequency mutations.

\section{Real-time polymerase chain reaction verification}

RT-PCR was used to verify and compare the expression levels of four mutated genes (RWDD4, SDHC, SEPT7, and $S F N$ ) between tumor tissues and adjacent non-tumor tissues. Total cellular RNA was extracted with $1 \mathrm{ml}$ TRIzol reagent (9109, TaKaRa), and RNA purity was detected using a microplate reader (Infinite M100 PRO, TECAN). One microgram of RNA was reverse-transcribed into cDNA using $4 \mu \mathrm{l} 5 \times$ PrimeScript RT Master MIX (RR036A, TAKARA). RT-PCR was conducted on the ABI Prism 7900HT Fast Real-Time PCR system using the specific primer pairs as described in Table 1 and SYBR Premix EX Taq (A25742, Thermo). The protocol was set as follows: $50^{\circ} \mathrm{C}$ for $2 \mathrm{~min}, 95^{\circ} \mathrm{C}$ for $10 \mathrm{~min}, 40$ cycles of $95^{\circ} \mathrm{C}$ for $10 \mathrm{~s}, 60^{\circ} \mathrm{C}$ for $30 \mathrm{~s}$. All the samples were normalized to the corresponding expression of internal control GAPDH. The test was performed in triplicate and the relative expression levels were calculated with the $2^{-\Delta \Delta} \mathrm{Ct}$ method. $p<0.05$ and $p<0.01$ respectively indicated a significant difference and extremely significant difference.

Table 1 Primers and primer sequences of Q-PCR genes

\begin{tabular}{ll}
\hline Primer & Sequence(5'-3') \\
\hline RWDD4-hF & TGCCAACGAGGACCAGGAG \\
RWDD4-hR & AGGCTTGGGATCACCATTा \\
SDHC-hF & TGCCTCCGAGCCCACTTIA \\
SDHC-hR & TTATTCCAGAACCGCTCCATC \\
SEPT7-hF & AGGAGCGTCAACAGCAGCAC \\
SEPT7-hR & CCCAATCCAGATTCACCCACT \\
SFN-hF & GCCGAACGCTATGAGGACA \\
SFN-hR & GCTCAATACTGGACAGCACCC \\
GAPDH-hF & TGACAACTTTGGATCGTGGAAGG \\
GAPDH-hR & AGGCAGGGATGATGTTCTGGAGAG \\
\hline
\end{tabular}

\section{Results}

Identification of DEGs and functional enrichment analysis As compared with the MCF10A cell line, 5391, 7073, and 7944 DEGs were identified in MCF10.DCIS, Sum102, and Sum22 cell lines, respectively, and the ratios of up- and downregulated DEGs were 0.98, 0.99, and 1.26, respectively, without any significant bias of up- or downregulation.

Subsequently, a functional enrichment analysis of upand downregulated DEGs in each DCIS cell line was performed. For the upregulated DEGs, seven abnormal GO terms were enriched, including positive regulation of tumor necrosis factor production, second-messengermediated signaling, response to endogenous stimulus, axon and neuron ensheathment, isoprenoid biosynthetic process, and regulation of action potential (Fig. 1a). The significance of these seven GO terms was different among the three cancer cell lines. For example, the enrichment of positively regulated tumor necrosis factor production was most significant in Sum102, while the enrichment of second-messenger-mediated signaling was more common in Sum225 and MCF10.DCIS cell lines.

For the downregulated DEGs, GO terms such as positive regulation of programmed cell death, RNA processing, and response to endogenous stimulus were enriched (Fig. 1b). However, only downregulated DEGs of Sum102 and Sum225 cell lines were enriched in translational elongation, as associated with the regulation of protein translation process.

\section{Identification of core DEGs and functional enrichment analysis}

Through VENN analysis, we detected 603 upregulated and 1043 downregulated DEGs overlapping in the three cancer cell lines (Fig. 2), including 16 upregulated TFs (e.g., HOXA2, HOXB2, HOXC10, and NKX2-1) and 36 downregulated TFs (e.g., $B A C H 1$ and $C D X 1$ ), respectively. Herein, these overlapping DEGs were considered as the core DEGs. The upregulated overlapping DEGs were significantly enriched in the regulation of cAMP biosynthetic process, response to lipopolysaccharide, small GTPase-mediated signal transduction, and cytokine production, while the downregulated overlapping DEGs were significantly enriched in the regulation of cell migration, negative regulation of cell proliferation, cell adhesion, and JNK cascade (Table 2).

According to the analysis of cellular localization, 11 core upregulated DEGs (e.g., FREM2 and NOD2) and 17 core downregulated DEGs (e.g., PTPRK and CAV1) were associated with the cell surface, suggesting that the characteristics of cell surface proteins of the DCIS cells could help distinguish DCIS cells from normal cells. Additionally, the protein products of 5 upregulated DEGs (LAMB4, COL9A2, FREM2, and COL1OA1) and 12 downregulated DEGs (e.g., COL17A1, 


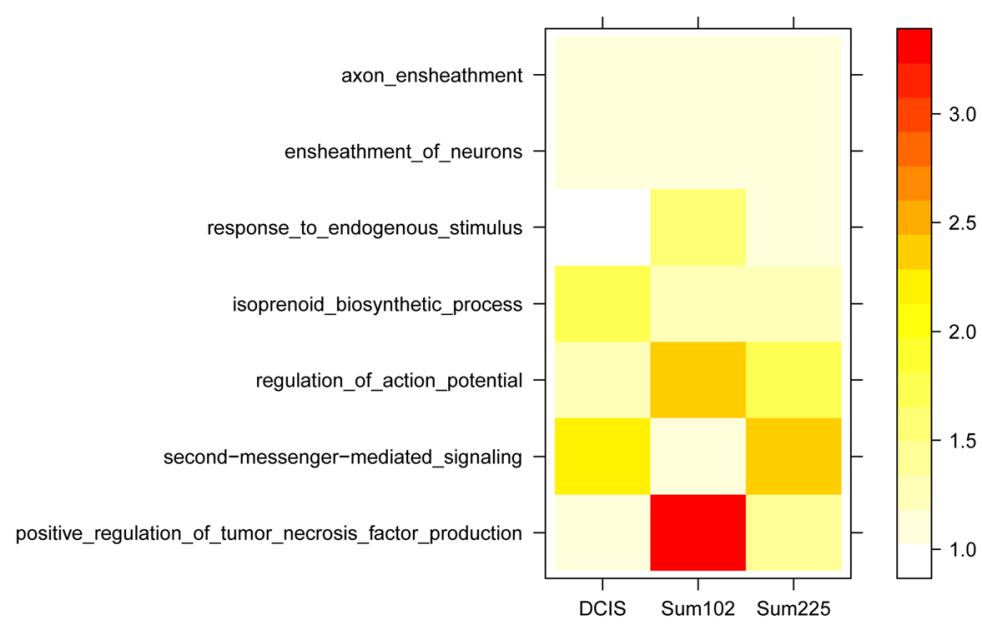

b

Common BP of down-regulated genes in all three cell-lines

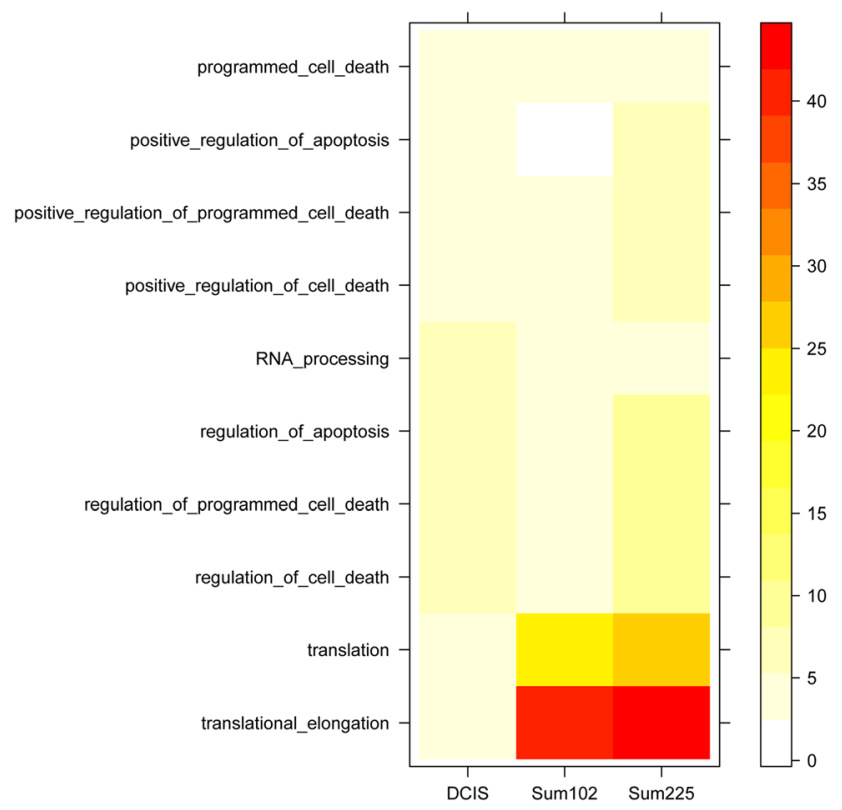

Fig. 1 Functional enrichment analysis of up- and downregulated genes in MCF10.DCIS, Sum102, and Sum225 cell lines. a Functional enrichment analysis of upregulated genes. $\mathbf{b}$ Functional enrichment analysis of downregulated genes. Colors in every cell represent the significance of functional enrichment in the cell lines. Significance is proportionate to color depth

COL7A1, and LAMA5) were specifically located in the ECM, indicating that DCIS might be diagnosed via the detection of specific protein factors in blood (Table 3).

Upstream TFs and functional enrichment analysis of DEGs According to motif scanning, six TF motifs each were enriched in the promoter regions of the core up- and downregulated DEGs (Table 4). Especially, CREB1 and E2F1 motifs were identified in the promoters of the upregulated overlapping DEGs.

\section{Detection of somatic mutations and SNVs}

In total, 1104, 2833, and 1132 somatic mutations were identified in MCF10.DCIS, Sum102, and Sum225 cell lines, respectively (Fig. 3a). For each cell line, transition was the main mutation type (>72\%), the frequency of transversion was approximately $25 \%$, and the frequency of insertion-deletion (indel) was only $3 \%$ (Fig. 3b). A total of 19, 31, and 17 indel sites were detected in MCF10.DCIS, Sum102, and Sum225 cell lines, respectively. Furthermore, four high-frequency mutant genes were identified according to the criteria in at least two cell lines (Table 5). Base 
a

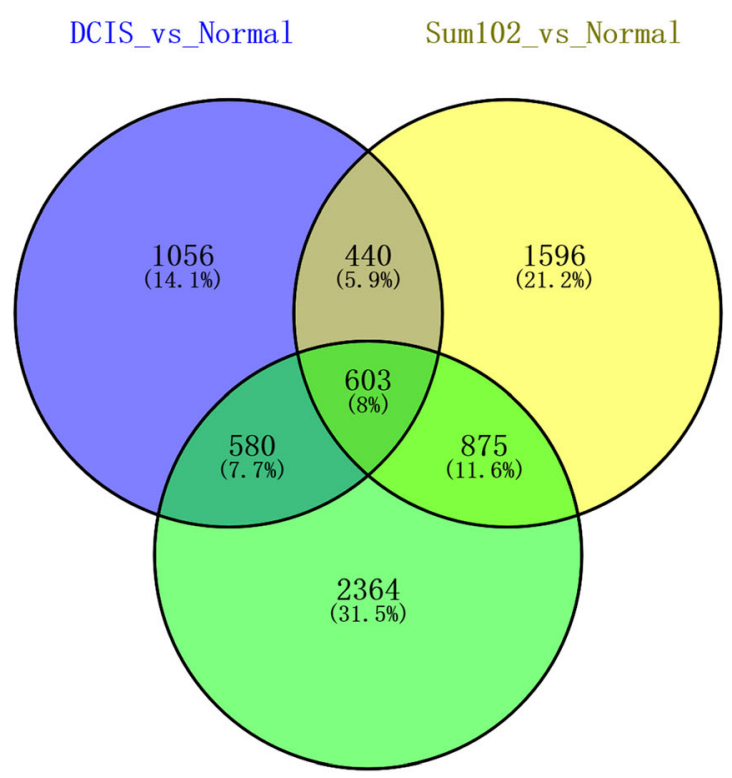

Sum225 vs Normal b

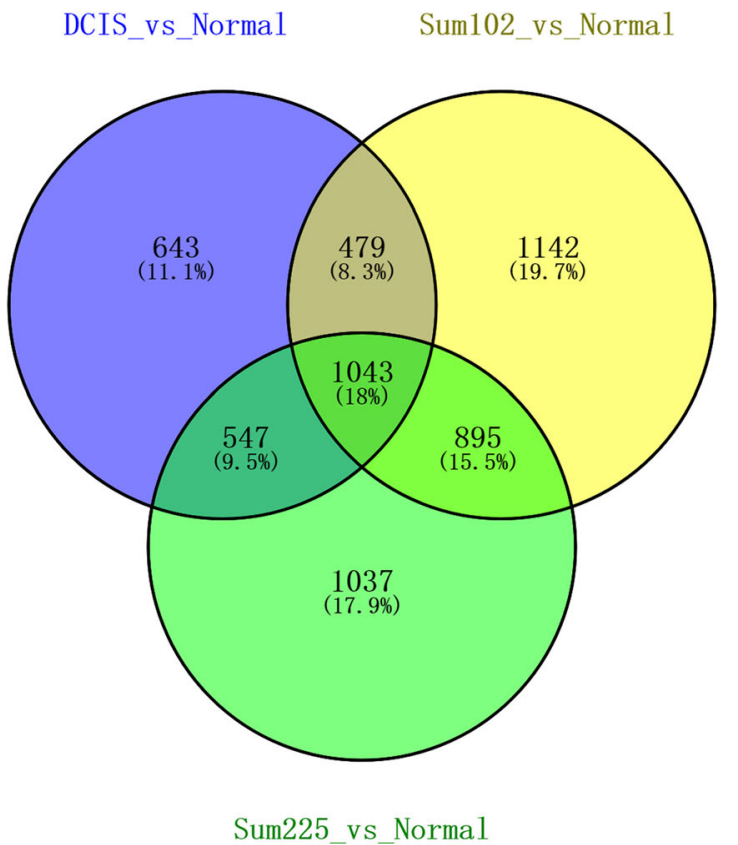

Fig. 2 VENN analysis of differentially co-expressed genes in MCF10.DCIS, Sum102, and Sum225 cell lines. a Upregulated DEGs in the MCF10.DCIS, Sum102, and Sum225 cell lines. b downregulated DEGs in the MCF10.DCIS, Sum102, and Sum225 cell lines.

mutations located in chr1:161332189 and chr1:27190350 led to missense mutations in SDHC and SFN, respectively. Base mutations in chr4:184562622 and chr7:35840881 were located at the exon splicing sites and led to an abnormal post-transcriptional splicing of RWDD4 and SEPT7. SDHC mutations occurred in Sum102 and Sum225 cell lines at the same mutant site, as well as SFN. RWDD4 and SEPT7 mutations occurred in both MCF10.DCIS and Sum102 cell lines.

\section{RT-PCR verification}

Consistent with the bioinformatics analysis results, the RT-PCR results revealed an overall downregulation. As shown in Fig. 4, the expression levels of RWDD4, SEPT7, and $S F N$ were significantly lower in the breast cancer tissues than in the adjacent non-tumor tissues $(p<0.05$ for RWDD4, $p<0.05$ for SEPT7, $p<0.01$ for SFN). Although the expression level of $S D H C$ was not significantly downregulated in the breast cancer tissues, the trend of $S D H C$ expression was consistent with that of RWDD4, SEPT7, and SFN expression.

\section{Discussion}

In this study, based on the RNA-seq data of DCIS, 603 core upregulated and 1043 core downregulated DEGs were identified among the three DCIS cell lines. Among them, there were 16 upregulated and 36 downregulated TFs.
Furthermore, the products of 11 upregulated and 17 downregulated DEGs were located on the cell surface, and the products of 5 upregulated and 12 downregulated DEGs were located in the ECM. In addition, six TF motifs each were enriched in the promoter regions of core up- and downregulated DEGs, respectively. Additionally, four high-frequency mutant genes were detected.

Among the core DEGs, the upregulated genes, such as HOXA2, HOXB2, HOXC10, and NKX2-1, were identified as TFs. HOXA2, HOXB2, and HOXC10 belong to the homeobox family of genes, which play an important role in morphogenesis and differentiation [21]. A previous study had reported that some homeobox genes, such as $H O X B 13, T L X 1$, and $H N F 1 B$, were hypermethylated in the early stages of breast cancer [22]. HOXB2 and $H O X C 10$ were identified to be differentially expressed in the hyperplastic enlarged lobular unit when compared with the normal terminal duct lobular unit, and other homeobox genes (e.g., HOXA4 and HOXB6) were differentially expressed in DCISs with respect to normal tissues [23]. In addition, DCIS is closely associated with the silencing of HOXA2 [23]. As a result, the homeobox genes $H O X A 2, H O X B 2$, and $H O X C 10$ may be closely correlated with DCIS. NKX2-1 (NK2 homeobox 1, also known as TITF1/TTF-1) is a thyroid-specific transcription factor, which regulates the expressions of thyroid-specific genes as well as genes involved in morphogenesis [24]. There is 
Table 2 Functional enrichment analysis of the overlapping DEGs

\begin{tabular}{|c|c|c|c|}
\hline GO Term & Gene Counts & TF Genes & $p$ value \\
\hline \multicolumn{4}{|l|}{ Downregulated overlapping DEGs } \\
\hline Translation & 38 & & $3.88 \mathrm{E}-06$ \\
\hline Positive regulation of programmed cell death & 41 & HIP1, ID3, IFI16, NR3C1, PML, RXRA, TP53BP2 & 1.36E-04 \\
\hline RNA processing & 46 & & 7.09E-04 \\
\hline Regulation of cell migration & 20 & & 8.03E-04 \\
\hline Negative regulation of cell proliferation & 33 & ESR2, NKX3-1, NME2, PML, RXRA & 0.001239 \\
\hline Regulation of lipid metabolic process & 15 & NR3C1, NR5A1, PPARA & 0.001380 \\
\hline Cell adhesion & 52 & LEF1, NME2 & 0.004558 \\
\hline JNK cascade & 9 & & 0.008755 \\
\hline Cellular protein localization & 33 & & 0.008962 \\
\hline Response to hormone stimulus & 30 & ESR2, PPARA, RXRA & 0.010205 \\
\hline \multicolumn{4}{|l|}{ Upregulated overlapping DEGs } \\
\hline Positive regulation of tumor necrosis factor production & 4 & & 0.005861 \\
\hline Regulation of tumor necrosis factor production & 5 & & 0.007442 \\
\hline Response to lipopolysaccharide & 7 & $N K X 2-1$ & 0.013341 \\
\hline Small GTPase mediated signal transduction & 15 & & 0.024022 \\
\hline Cytokine production & 5 & & 0.028739 \\
\hline Regulation of phosphate metabolic process & 20 & & 0.040473 \\
\hline Regulation of cAMP biosynthetic process & 7 & & 0.043362 \\
\hline Blood circulation & 10 & & 0.047845 \\
\hline
\end{tabular}

$D E G s$ differentially expressed genes, $T F$ transcription factor, GO gene ontology

evidence that TTF-1 stain is negative in the DCIS cells [25], suggesting that $N K X 2-1$ may play a crucial role.

Furthermore, the protein encoded by FREM2 (FRAS1 related extracellular matrix protein 2) was detected to be upregulated on the cell surface and in the ECM of DCIS tissue. FREM2, an integral membrane protein, plays a role in epidermal-dermal interactions [26]. FREM2 has been identified as a target gene of TFAP2C (transcription factor AP-2 gamma) in hormone-responsive breast cancer cells [27]. However, no study has reported the association of FREM2 with DCIS so far. Thus, we speculated that FREM2 may play a pivotal role in DCIS, by participating in cell interactions.

Based on the motif scanning, E2F1 was identified as an upstream TF for the core downregulated DEGs. E2F1 (E2F transcription factor 1) is a target of c-Myc that promotes the cell cycle [28]. It has been demonstrated that E2F-1 expression is significantly higher in DCIS than in the normal breast tissue [29]. Moreover, E2F1 is involved in
ARF tumor suppressor expression and activation in DCIS [30, 31]. Collectively, the dysfunction of the transcriptional regulatory pathway of $E 2 F 1$ may contribute to cell multiplication in DCIS. Additionally, the CREB1 (cAMP response element-binding protein 1) motif was identified in the promoter regions of the upregulated overlapping DEGs. CREB1 belongs to a subfamily of the leucine zipper with basic domain family of cellular transcription factors. This gene has been detected to be expressed at a high level in breast cancer cell lines MCF7 and MDA-MB-231 and is linked to patients' overall and disease-free survival [32]. Therefore, CREB1 may participate in the progression of DCIS via regulation of downstream genes.

In the present study, four high-frequency mutant genes (RWDD4, SDHC, SEPT7 and SFN) were identified. RWDD4 (RWD domain-containing protein 4) is a member of the RWD domain protein superfamily

Table 3 The overlapping DEGs associated with cell surface and extracellular matrix part

\begin{tabular}{|c|c|c|c|c|}
\hline \multirow[t]{2}{*}{ Location } & \multicolumn{2}{|c|}{ Upregulated DEGs } & \multicolumn{2}{|c|}{ Downregulated DEGs } \\
\hline & Count & Gene name & Counts & Gene name \\
\hline Cell surface & 11 & $\begin{array}{l}\text { NOD2, IL12RB1, CORIN, FREM2, FLT3, LRRTM1, } \\
\text { FCER1G, CHRNA4, TPO, HSPA5, SCNN1A }\end{array}$ & 17 & $\begin{array}{l}\text { PTPRK, CAV1, PARD3, SELL, LHCGR, MFGE8, ITGA4, ADA, CDH13, } \\
\text { SDC1, BGN, SULF2, RC3H2, HSPB1, TGFBR3, SCNN1G, ADAM9 }\end{array}$ \\
\hline $\begin{array}{l}\text { Extracellular } \\
\text { matrix part }\end{array}$ & 5 & LAMB4, COL9A2, FREM2, COL11A2, COL10A1 & 12 & $\begin{array}{l}\text { COL17A1, COL7A1, LAMA5, NID1, COL1A1, LAMB1, DST, PRSS12, } \\
\text { COL4A6, COL4A5, FN1, ANXA2 }\end{array}$ \\
\hline
\end{tabular}


Table 4 Upstream TF analysis of overlapping DEGs

\begin{tabular}{lcc}
\hline Promoter regions & Candidate TF counts & TF genes \\
\hline Downregulated DEGs & 6 & E2F1, ELK1, HIF1A, MYB, NR2C2, TFAP2A \\
Upregulated DEGs & 6 & CREB1, ELK4, GABPA, NFYA, PAX5, ZBTB7B
\end{tabular}

$D E G s$ differentially expressed genes $T F$ transcription factor

[33]. RWDD4 contains a RWD domain that is involved in protein-protein interactions [34]. The knockdown of RWDD4 inhibits transitional cell carcinoma (TCC), cell proliferation, migration, and invasion [35]. However, no study has revealed RWDD4 mutations in DCIS so far. SDHC encodes one of the four nuclear-encoded subunits that comprise succinate dehydrogenase, a mitochondrial protein. SDHC mutation has been confirmed to be associated with various cancers, such as kidney cancer [36], gastrointestinal stromal tumors [37], pheochromocytoma [38], and head and neck paraganglioma [39]. There is no evidence that $S D H C$ mutation is linked to DCIS, although studies have shown the association of $S D H C$ homologs with breast cancer. $S D H B$ and $S D H D$ variants are highly related to the increased prevalence of breast cancers [40]. Furthermore, a previous study has reported loss of $S D H A$ or $S D H B$ expression in $3 \%$ of breast cancers [41]. The study also suggested that the downregulation of SDHC promoted epithelial to mesenchymal transition, which was accompanied by the structural remodeling of mitochondrial organelles. This may confer a survival benefit upon exposure to a harmful microenvironment during cancer progression [42]. Therefore, we speculated that the $S D H C$ mutation might be closely related to the progression of DCIS. SEPT7 encodes septin 7, which is highly similar to the CDC10 protein of Saccharomyces cerevisiae [43]. There has been no evidence to support the relationship between SEPT7 and DCIS so far, while DNA methylationinduced altered expression of SEPT9 has been observed during breast tumorigenesis [44]. The study revealed that SEPT2 and SEPT7 play an important role in cell migration and invasion in breast cancers by controlling the activation of MEK/ ERK MAPKs, and that targeting septin proteins may provide

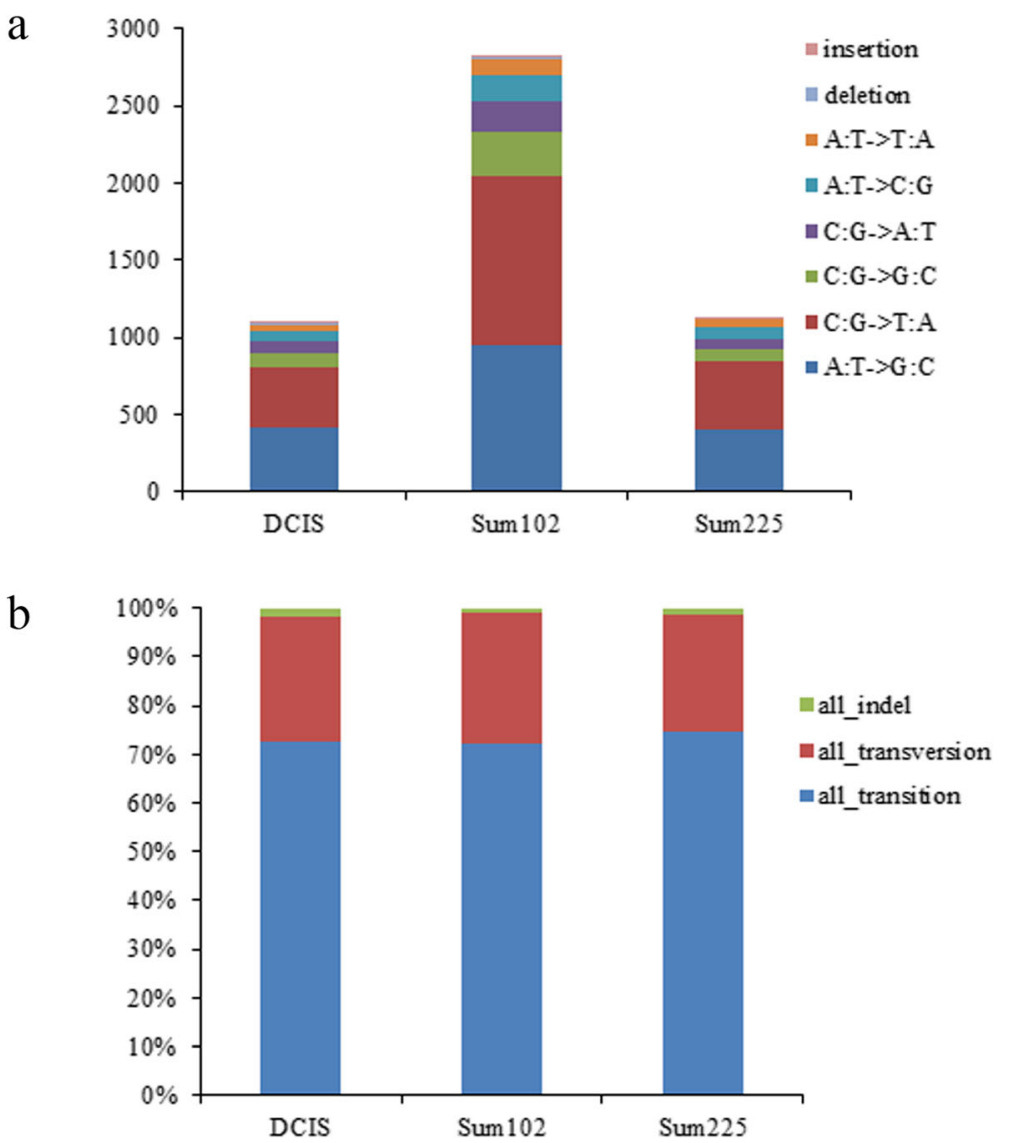

Fig. 3 Statistics of single nucleotide variations in MCF10.DCIS, Sum102, and Sum225 cell lines. a Total number of various mutations in samples. b Percentages of various mutations in samples 
Table 5 Mutant sites with high-frequency in breast ductal carcinoma in situ cell lines

\begin{tabular}{lllllll}
\hline Chromosome & Position & Reference base & Mutated base & Corresponding gene & Observed frequency & Tumor-associated genes \\
\hline chr1 & 161332189 & C & A & SDHC & 2 & Tumor suppressor \\
chr1 & 27190350 & G & A & SFN & 2 & 2 \\
chr4 & 184562622 & C & A & RWDD4 & 2 & \\
chr7 & 35840881 & G & C & SEPT7 & 2 & \\
\hline
\end{tabular}

a new direction for breast cancer treatment [45]. Thus, SEPT7 might be involved in DCIS. SFN (stratifin, also known as 14-3-sigma) has been found to be hypermethylated and related to cell-cycle regulation in human breast cancer cells [46]. The previous study by Li et al. demonstrated that isothiocyanate SFN treatment inhibited DCIS stem-like cells in vivo and in vitro [47]. Li et al. reported that SFN treatment reprogrammed DCIS stem-like cells, as evidenced by the significant changes in exosomal secretion, which is more closely resembling that of the non-stem cancer cells [48]. Therefore, SFN might be involved in DCIS.

\section{Conclusions}

In conclusion, differentially expressed TFs (e.g., $H O X A 2, H O X B 2, H O X C 10$, and NKX2-1), genes that encode proteins located on the cell surface and in the ECM of DCIS (e.g., FREM2), and TFs of DEGs (e.g., $E 2 F 1$ and $C R E B 1)$, as well as high-frequency mutant genes (RWDD4,SDHC, SEPT7, and SFN), may participate in the progression of DCIS. These findings may contribute to a better understanding of the molecular mechanism underlying DCIS.

\section{Abbreviations}

ALDH5A1: Aldehyde dehydrogenase 5A1; BDC: Breast ductal carcinoma; BP: Biological process; CC: Cellular component; DAVID: The Database for Annotation, Visualization and Integrated Discovery; DCIS: Ductal carcinoma in situ; DEGs: Differentially expressed genes; GEO: Gene Expression Omnibus; IDC: Invasive ductal carcinomas; MF: Molecular function; NCBI: National Center for Biotechnology Information; SNPs: Single nucleotide polymorphisms; SNVs: Single nucleotide variations; TAGs: Tumor-associated genes; TF: Transcription factor; TSGs: Tumor suppressor genes
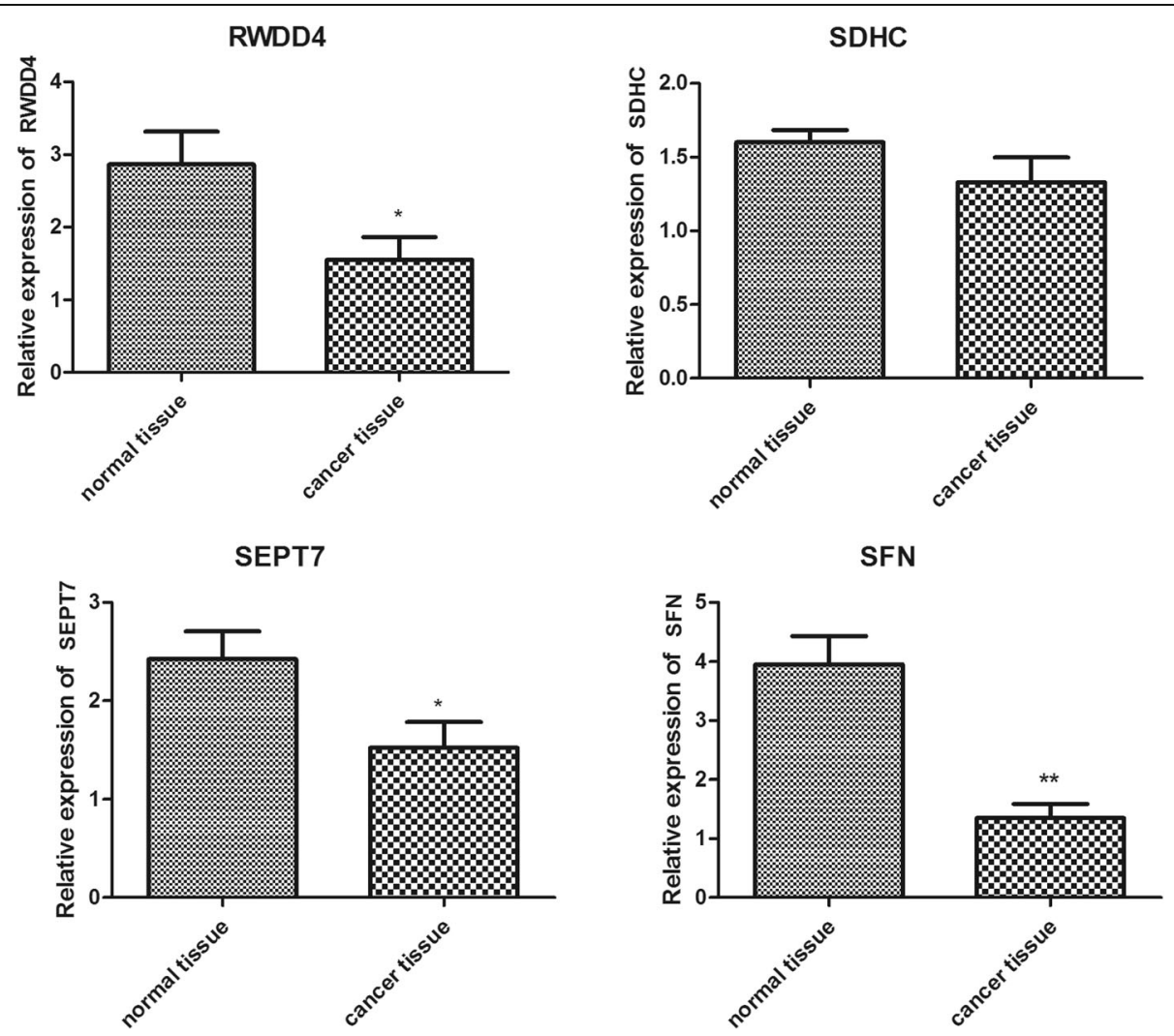

Fig. 4 Verification results of real-time polymerase chain reaction (RT-PCR) of RWDD4, SDHC, SEPT7, and SFN. * Indicates a significant difference between normal tissue and cancer tissue $(p<0.05)$. ${ }^{*}$ Indicates an extremely significant difference between normal tissue and cancer tissue $(p<0.01)$ 


\section{Acknowledgements}

None.

\section{Authors' contributions}

CZ conceived the research, acquired the data and drafted the manuscript. $\mathrm{HH}, \mathrm{JS}, \mathrm{JW}$, and KW analyzed and interpreted the data. JL revised the paper for important content. All authors approved the final paper.

\section{Funding}

No funding was received.

\section{Availability of data and materials}

The datasets used and/or analyzed during the current study are available from the corresponding author on reasonable request.

\section{Ethics approval and consent to participate}

Not applicable.

\section{Consent for publication}

Not applicable.

\section{Competing interests}

The authors declare that they have no competing interests.

\section{Received: 6 June 2019 Accepted: 18 February 2020}

Published online: 10 March 2020

\section{References}

1. Bateman NW, Sun M, Bhargava R, Hood BL, Darfler MM, Kovatich AJ, Hooke JA, Krizman DB, Conrads TP. Differential proteomic analysis of late-stage and recurrent breast cancer from formalin-fixed paraffin-embedded tissues. J Proteome Res. 2010:10:1323-32.

2. DeSantis CE, Ma J, Goding Sauer A, Newman LA, Jemal A. Breast cancer statistics, 2017, racial disparity in mortality by state. CA Cancer J Clin. 2017; 67:439-48

3. Virnig BA, Tuttle TM, Shamliyan T, Kane RL. Ductal carcinoma in situ of the breast: a systematic review of incidence, treatment, and outcomes. J Natl Cancer Inst. 2010;102:170-8.

4. Elias EV, de Castro NP, Pineda PHB, Abuázar CS, de Toledo Osorio CAB, Pinilla MG, da Silva SD, Camargo AA, Silva WA Jr, Ferreira ENE Jr. Epithelial cells captured from ductal carcinoma in situ reveal a gene expression signature associated with progression to invasive breast cancer. Oncotarget. 2016;7:75672.

5. Ebata A, Suzuki T, Takagi K, Miki Y, Onodera $Y$, Nakamura $Y$, Fujishima F, Ishida K, Watanabe M, Tamaki K. Abstract P6-05-14: Estrogen-induced genes in ductal carcinoma in situ (DCIS): their comparison with invasive ductal carcinoma. Cancer Res. 2012;72 P6-05-14-P06-05-14.

6. Lee S, Stewart S, Nagtegaal I, Luo J, Wu Y, Colditz G, Medina D, Allred DC. Differentially expressed genes regulating the progression of ductal carcinoma in situ to invasive breast cancer. Cancer Res. 2012;72:4574-86.

7. Mazzola E, Cheng S-C, Parmigiani G. The penetrance of ductal carcinoma in situ among BRCA1 and BRCA2 mutation carriers. Breast Cancer Res Treat. 2013;137:315-8.

8. Sakr RA, Martelotto LG, Baslan T, Ng CK, Kendall J, Rodgers L, Cox H, Riggs M, D'Itali S, Stepansky A. Intra-tumor heterogeneity and clonal changes in the progression of DCIS to invasiveness: Combined tumor bulk and single cell analysis. Cancer Res. 2015;75:2989.

9. Abba MC, Gong T, Lu Y, Lee J, Zhong Y, Lacunza E, Butti M, Takata Y, Gaddis S, Shen J. A Molecular Portrait Of High-Grade Ductal Carcinoma In Situ (DCIS). Cancer Res. 2015; canres. 0506.2015.

10. Tian X, Han Y, Yu L, Luo B, Hu Z, Li X, Yang Z, Wang X, Huang W, Wang H. Decreased expression of ALDH5A1 predicts prognosis in patients with ovarian cancer. Cancer Biol Ther. 2017;18:245-51.

11. Kaur H, Mao S, Li Q, Sameni M, Krawetz SA, Sloane BF, Mattingly RR. RNA-Seq of human breast ductal carcinoma in situ models reveals aldehyde dehydrogenase isoform $5 \mathrm{~A} 1$ as a novel potential target. PloS One. 2012;7:e50249.

12. Trapnell C, Pachter L, Salzberg SL. TopHat: discovering splice junctions with RNA-Seq. Bioinformatics. 2009;25:1105-11.

13. Trapnell C, Williams BA, Pertea G, Mortazavi A, Kwan G, van Baren MJ, Salzberg SL, Wold BJ, Pachter L. Transcript assembly and quantification by
RNA-Seq reveals unannotated transcripts and isoform switching during cell differentiation. Nature Biotechnol. 2010;28:511-5.

14. Tarazona S, García-Alcalde F, Dopazo J, Ferrer A, Conesa A. Differential expression in RNA-seq: a matter of depth. Genome Res. 2011;21:2213-23.

15. Da Wei Huang BTS, Lempicki RA. Systematic and integrative analysis of large gene lists using DAVID bioinformatics resources. Nat Protocols. 2008:4:44-57.

16. Zhao M, Kim P, Mitra R, Zhao J, Zhao Z. TSGene 2.0: an updated literaturebased knowledgebase for tumor suppressor genes. Nucleic Acids Res. 2015; 44:D1023-31.

17. Chen J-S, Hung W-S, Chan H-H, Tsai S-J, Sun HS. In silico identification of oncogenic potential of fyn-related kinase in hepatocellular carcinoma. Bioinformatics. 2013;29:420-7.

18. He HH, Meyer CA, Shin H, Bailey ST, Wei G, Wang Q, Zhang Y, Xu K, Ni M, Lupien M. Nucleosome dynamics define transcriptional enhancers. Nat Genet. 2010:42:343-7.

19. McKenna A, Hanna M, Banks E, Sivachenko A, Cibulskis K, Kernytsky A, Garimella K, Altshuler D, Gabriel S, Daly M. The Genome Analysis Toolkit: a MapReduce framework for analyzing next-generation DNA sequencing data. Genome Res. 2010;20:1297-303.

20. Cheng Y-C, Hsiao F-C, Yeh E-C, Lin W-J, Tang C-YL, Tseng H-C, Wu H-T, Liu $\mathrm{C}-\mathrm{K}$, Chen $\mathrm{C}-\mathrm{C}$, Chen $\mathrm{Y}-\mathrm{T}$. VarioWatch: providing large-scale and comprehensive annotations on human genomic variants in the next generation sequencing era. Nucleic Acids Res. 2012;40:W76-81.

21. SuryaDeva $S$, Khan MB. Role of homeobox genes in tooth morphogenesis: a review. J Clin Diagn Res. 2015;9:ZE09.

22. Tommasi S, Karm DL, Wu X, Yen Y, Pfeifer GP. Methylation of homeobox genes is a frequent and early epigenetic event in breast cancer. Breast Cancer Res. 2009:11:R14.

23. Coradini D, Boracchi P, Oriana S, Biganzoli E, Ambrogi F. Epithelial cell identity in hyperplastic precursors of breast cancer. Chin J Cancer. 2014

24. Fernandez LP, Lopez-Marquez A, Santisteban P. Thyroid transcription factors in development, differentiation and disease. Nat Rev Endocrinol. 2015;11:29.

25. Ni YB, Tsang JY, Shao MM, Chan SK, Tong J, To KF, Tse GM. TTF-1 expression in breast carcinoma: an unusual but real phenomenon. Histopathology. 2014;64:504-11.

26. Timmer JR, Mak TW, Manova K, Anderson KV, Niswander L. Tissue morphogenesis and vascular stability require the Frem2 protein, product of the mouse myelencephalic blebs gene. Proc Natl Acad Sci U S A. 2005;102: 11746-50.

27. Woodfield GW, Chen Y, Bair TB, Domann FE, Weigel RJ. Identification of primary gene targets of TFAP2C in hormone responsive breast carcinoma cells. Genes Chromosomes Cancer. 2010;49:948-62.

28. Gordan JD, Bertout JA, Hu C-J, Diehl JA, Simon MC. HIF-2a promotes hypoxic cell proliferation by enhancing c-myc transcriptional activity. Cancer Cell. 2007:11:335-47.

29. Zhang SY, Liu SC, Al-Saleem LF, Holloran D, Babb J, Guo X, Klein-Szanto AJ. E2F-1: a proliferative marker of breast neoplasia. Cancer Epidemiol Biomarkers Prev. 2000;9:395-401.

30. Zindy F, Williams RT, Baudino TA, Rehg JE, Skapek SX, Cleveland JL, Roussel MF, Sherr CJ. Arf tumor suppressor promoter monitors latent oncogenic signals in vivo. Proc Natl Acad Sci. 2003;100:15930-5.

31. Vestey S, Sen C, Calder C, Perks C, Pignatelli M, Winters Z. p14ARF expression in invasive breast cancers and ductal carcinoma in situ-relationships to p53 and $\mathrm{Hdm} 2$. Breast Cancer Res. 2004:6:R571-85.

32. Chhabra A. Molecular insight of the CAMP Responsive Element Binding Protein (CREB) in human breast cancer: Cardiff University; 2012.

33. Doerks T, Copley RR, Schultz J, Ponting CP, Bork P. Systematic identification of novel protein domain families associated with nuclear functions. Genome Res. 2002:12:47-56.

34. Winter JM, Gildea DE, Andreas JP, Gatti DM, Williams KA, Lee M, Hu Y, Zhang S, Mullikin JC, Wolfsberg TG. Mapping complex traits in a diversity outbred F1 mouse population identifies germline modifiers of metastasis in human prostate cancer. Cell Syst. 2017:4:31-45 e36.

35. Hou Y. MiR-506 inhibits cell proliferation, invasion, migration and epithelialto-mesenchymal transition through targeting RWDD4 in human bladder cancer. Oncol Lett. 2019;17:73-8.

36. Ricketts CJ, Shuch B, Vocke CD, Metwalli AR, Bratslavsky G, Middelton L, Yang Y, Wei M-H, Pautler SE, Peterson J. Succinate dehydrogenase kidney cancer: an aggressive example of the Warburg effect in cancer. J Urology. 2012;188:2063-71. 
37. Killian JK, Miettinen M, Walker RL, Wang Y, Zhu YJ, Waterfall JJ, Noyes N, Retnakumar P, Yang Z, Smith WI. Recurrent epimutation of SDHC in gastrointestinal stromal tumors. Sci Translat Med. 2014;6:268ra177.

38. Weber A, Hoffmann MM, Neumann HP, Erlic Z. Somatic mutation analysis of the SDHB, SDHC, SDHD, and RET genes in the clinical assessment of sporadic and hereditary pheochromocytoma. Horm Cancer. 2012;3:187-92.

39. Ghayee HK, Isaacson B, Farley M, Click A, Pacak K, Shay JW. Unusual Case of a Hormone-Secreting SDHC Head and Neck Paraganglioma. In: Pancreas. Philadelphia: Lippincott Williams \& Wilkins; 2014. p. 496.

40. Ni Y, He X, Chen J, Moline J, Mester J, Orloff MS, Ringel MD, Eng C. Germline $\mathrm{SDHx}$ variants modify breast and thyroid cancer risks in Cowden and Cowden-like syndrome via FAD/NAD-dependant destabilization of p53. Hum Mol Genet. 2012;21:300-10

41. Kim S, Kim DH, Jung W-H, Koo JS. Succinate dehydrogenase expression in breast cancer. Springer Plus. 2013;2:299.

42. Røsland GV, Dyrstad SE, Tusubira D, Helwa R, Tan TZ, Lotsberg ML, Pettersen IKN, Berg A, Kindt C, Hoel F, et al. Epithelial to mesenchymal transition (EMT) is associated with attenuation of succinate dehydrogenase (SDH) in breast cancer through reduced expression of SDHC. Cancer Metab. 2019;7.

43. Nakatsuru S, Sudo K, Nakamura Y. Molecular cloning of a novel human CDNA homologous to CDC10 in Saccharomyces cerevisiae. Biochem Biophys Res Commun. 1994;202:82-7.

44. Connolly D, Yang Z, Castaldi M, Simmons N, Oktay MH, Coniglio S, Fazzari MJ, Verdier-Pinard P, Montagna C. Septin 9 isoform expression, localization and epigenetic changes during human and mouse breast cancer progression. Breast Cancer Res. 2011;13:R76.

45. Zhang N, Liu L, Fan N, Zhang Q, Wang W, Zheng M, Ma L, Li Y, Shi L. The requirement of SEPT2 and SEPT7 for migration and invasion in human breast cancer via MEK/ERK activation. Oncotarget. 2016;7:61587-600.

46. Jovanovic J, Rønneberg JA, Tost J, Kristensen V. The epigenetics of breast cancer. Mol Oncol. 2010;4:242-54

47. Li Q, Yao Y, Eades G, Liu Z, Zhang Y, Zhou Q. Downregulation of miR-140 promotes cancer stem cell formation in basal-like early stage breast cancer. Oncogene. 2014;33:2589-600

48. Li Q, Eades G, Yao Y, Zhang Y, Zhou Q. Characterization of a stem-like subpopulation in basal-like ductal carcinoma in situ (DCIS) lesions. J Biol Chem. 2014;289:1303-12.

\section{Publisher's Note}

Springer Nature remains neutral with regard to jurisdictional claims in published maps and institutional affiliations.

Ready to submit your research? Choose BMC and benefit from:

- fast, convenient online submission

- thorough peer review by experienced researchers in your field

- rapid publication on acceptance

- support for research data, including large and complex data types

- gold Open Access which fosters wider collaboration and increased citations

- maximum visibility for your research: over $100 \mathrm{M}$ website views per year

At $\mathrm{BMC}$, research is always in progress.

Learn more biomedcentral.com/submissions 